\title{
Higher physical activity is associated with increased androgens, low interleukin 6 and less aortic calcification in peripheral obese elderly women
}

\author{
R H Straub, L B Tankó ${ }^{1}$, C Christiansen ${ }^{1}$, P J Larsen ${ }^{1}$ and D S Jessop ${ }^{2}$ \\ Laboratory of Neuroendocrino-Immunology, Department of Internal Medicine I, University Hospital, 93042 Regensburg, Germany \\ ${ }^{1}$ Center for Clinical and Basic Research, 2750 Ballerup, Denmark \\ ${ }^{2}$ Henry Wellcome Laboratories for Integrative Neuroscience and Endocrinology, University of Bristol, Bristol BS1 3NY, UK \\ (Correspondence should be addressed to R H Straub; Email: rainer.straub@klinik.uni-regensburg.de)
}

\begin{abstract}
The presence of peripheral fat mass (PFM) appears to counteract the atherogenic trends of central fat mass through mechanisms presently poorly understood. In elderly women with distinct forms of body fat distribution, we wanted to study whether physical activity and aortic calcification are related to plasma levels of cortisol, $17-\alpha$-hydroxyprogesterone (17- $\alpha$-OHP), dehydroepiandrosterone (DHEA), androstenedione (ASD), and interleukin 6 (IL6) accomplishing an antiatherogenic milieu. A total of 276 well-defined generally healthy women aged $60-85$ years were included. Categorization of body fat distribution was based on the relative presence of central to PFM measured by dual-energy X-ray absorptiometry. Women meticulously reported weekly physical activity. Outcome measures were aortic calcification

between lumbar vertebra L1 and L4, plasma levels of hormones, and IL6. In peripheral adipose women, plasma DHEA and ASD increased with the degree of physical activity. This was also mirrored in the ratios of cortisol/ DHEA and cortisol/17- $\alpha$-OHP. Peripheral adipose women with high DHEA relative to cortisol had less severe aortic calcification, and in the same group a higher level of physical activity was associated with lower levels of plasma IL6. In conclusion, this study demonstrates that high physical activity is associated with a high circulating androgen to cortisol ratio, low IL6, and less severe aortic calcification. Since androgens inhibit IL6 secretion, the activity-induced increase of these hormones might be an anti-atherogenic signal.

Journal of Endocrinology (2008) 199, 61-68
\end{abstract}

\section{Introduction}

The insulin resistance syndrome is an established risk factor for macrovascular disease and type 2 diabetes, two important health problems among the elderly (more than 65 years; Raji \& Plutzky 2002, Grant \& Meigs 2004). The presence of insulin resistance syndrome is a function of body fat distribution rather than body mass index (BMI; Tanko et al. 2003). It has been demonstrated that peripheral adiposity confers protection against diabetes and atherosclerosis in elderly people (Tanko et al. 2003, Ferreira et al. 2004, Snijder et al. 2004). This protective association is mediated through mechanisms involving insulin sensitization, which was not observed in central adipose women with a low percentage of peripheral fat mass (PFM; Tanko et al. 2003). Factors of this protective association are gradually being discovered but still remain poorly understood (Tanko et al. 2004). The role of hormonal factors might be important because different response patterns to hormonal stimuli with an increased hypothalamic-pituitaryadrenal axis activity have been discovered in peripheral versus central adipose people (Vicennati \& Pasquali 2000).

Apart from cortisol, androgens have been linked to atherogenesis and insulin resistance (Nestler et al. 1992, Hautanen et al. 1994, Phillips et al. 1997). However, the relation between androgens, particularly dehydroepiandrosterone (DHEA), and atherosclerosis still remains an open question (Wu \& von 2003). Since adrenal androgens such as DHEA and androstenedione (ASD) are able to inhibit proinflammatory cytokines from different cell types such as leukocytes (Straub et al. 1998, Kipper-Galperin et al. 1999, Gordon et al. 2001, Kim et al. 2006), inclusion of cytokines such as interleukin 6 (IL6) might be an additional important cofactor that determines the role of androgens in obesityrelated atherosclerosis and insulin resistance syndrome. In addition, the level of physical activity also determines the risk for atherosclerosis and insulin resistance syndrome (Laukkanen et al. 2002, Tanko et al. 2003). However, the complex interactions between level of physical activity, body fat distribution, adrenal androgens, and IL6 have not been investigated in a systematic manner.

Therefore, the purpose of this study was to investigate associations of self-reported physical activity with plasma 
levels of cortisol, adrenal androgens, and IL6 in postmenopausal women with distinct forms of body fat distribution.

\section{Patients and methods}

\section{Subjects and blood samples}

The study population consisted of 276 well-defined generally healthy women, 60-85 years old, who were selected from a large population-based cohort based on their distinct forms of body fat distribution. The method of selection has been described earlier in detail (Tanko et al. 2003). Briefly, central fat mass (sum of s.c. and visceral fat mass of the trunk) and PFM (fat mass of legs and arms) of the entire cohort $(n=1356$ subjects) were measured by dual-energy X-ray absorptiometry (Hologic QDR 4500). Fat deposits were expressed as the percentage of total body soft tissue mass and were scored on a scale from 1 to 4 (1: $<25$ th; $2: 25$ to 50 th; $3: 50$ to 75 th; and 4: $>75$ th percentile). Scores could be combined in $4 \times 4$ ways, of which 1 to 1 indicated lean women; 1 to 4 , women with peripheral obesity; 4 to 1 , women with central obesity; and 4 to 4 , women with general obesity (Tanko et al. 2003). Women with ongoing hormone replacement therapy $(n=10)$ were excluded from further analyses. All participants signed an approved consent form, and the study was carried out in accordance with the Helsinki Declarations. The Ethics Committee of Copenhagen County, Denmark, approved the study protocol.

Information about traditional cardiovascular factors were recorded: BMI, blood pressure, smoking habits, daily coffee consumption, regular alcohol consumption, and weekly physical activities of at least $1 \mathrm{~h}$ duration per occasion (1, none; 2 , once; 3 , twice; 4 , more than twice). Insulin resistance was estimated by the homeostasis model assessment of insulin resistance $\left(\mathrm{HOMA}_{\mathrm{IR}}\right)$ index (fasting insulin $(\mu \mathrm{U} / \mathrm{ml}) \times$ fasting glucose $(\mathrm{mmol} / \mathrm{l}))$ divided by $22 \cdot 5)$. All analyses were performed on plasma samples from fasting subjects taken between 0800 and $1000 \mathrm{~h}$. The basic characteristics of the patients are given in Supplementary Table 1, see Supplementary data in the online of version of the Journal of Endocrinology at http://joe.endocrinology-journals.org/ content/vol199/issue1/.

\section{Aortic calcification}

Aorta calcification is a direct surrogate of the atherosclerotic burden and an independent predictor of cardiovascular mortality in postmenopausal women (Wilson et al. 2001, Tanko et al. 2003, 2004, 2005). The severity of aorta calcification was graded using a semi-quantitative scoring system first introduced by the Framingham study group (Kauppila et al. 1997). Briefly, calcified deposits in the lumbar aorta adjacent to each lumbar vertebra between L1 and L4 were assessed separately for the anterior and posterior wall of the aorta using the midpoint of the intervertebral space as the boundary. Each wall of each segment was graded for the presence of calcified deposits with a score from 0 to 3 (0: no deposits, 1: less than $1 / 3$ of the aortic wall, $2: 1 / 3$ to $2 / 3$ of the aortic wall, 3 : more than $2 / 3$ of the aortic wall covered with calcified deposits). The sum of the scores of individual aortic segments both for the anterior and posterior walls, termed as anterior-posterior severity score, was used to describe the overall severity of vascular calcification in the lumbar aorta. The maximum score possibly given was $4 \times 2 \times 3=24$. The same investigator, who was blinded for all other results of the individual participants, carried out the image scoring. Intrarater correlations were in the range of $r=0 \cdot 92-0 \cdot 98$, similar to published results (Kauppila et al. 1997).

\section{Laboratory parameters (adrenal steroids and IL6)}

Several adrenal hormones were considered in order to detect major adrenal pathways of steroidogenesis (Fig. 1A). We used radioimmunometric assays for the quantitative determination of plasma levels of cortisol (Coulter Immunotech, Marseilles, France; detection limit: $10 \mathrm{nmol} / \mathrm{l})$. Plasma levels of 17- $\alpha$-hydroxyprogesterone (IBL, Hamburg, Germany; detection limit: $0.3 \mathrm{nmol} / \mathrm{l}$ ), DHEA (Diagnostic Systems Laboratory, Webster, TX, USA; detection limit: $0 \cdot 13 \mathrm{nmol} / \mathrm{l}$ ), and ASD (IBL; detection limit: $0.3 \mathrm{nmol} / \mathrm{l}$ ) were measured by means of immunometric enzyme immunoassays. Plasma levels of IL6 (high sensitivity Quantikine, R\&D Systems, Minneapolis, MN, USA; detection limit: $0 \cdot 2 \mathrm{pg} / \mathrm{ml}$ ) were measured using the same technique. Intraassay and interassay coefficients of variation for all above-mentioned tests were below $10 \%$.

\section{Calculation of hormone/hormone ratios}

In order to demonstrate the shift from one hormone to another hormone (compare Fig. 1A), the molar ratio of these hormones was calculated (given without unit, similar as compared with refs (Anderson \& Yen 1976, Barone et al. 1979, Straub et al. 2003)). This procedure detects a hormonal shift through one or more enzyme steps, which can demonstrate a preponderance of a certain hormonal pathway: cortisol/17- $\boldsymbol{\alpha}$-hydroxyprogesterone $(17-\alpha-\mathrm{OHP})$ for the pathway through CYP21A1P and CYP11B1 into the direction of cortisol, ASD/17- $\alpha$-OHP for the 17,20lyase (second reaction of the CYP17A1) into the direction of ASD, ASD/DHEA for the hydroxy- $\delta-5$-steroid dehydrogenase, $3 \beta$ - and steroid $\delta$-isomerase 1 (HSD3B1) into the direction of ASD, and cortisol/DHEA for the combined pathway through CYP21A1P, CYP11B1, 17,20lyase (second reaction of the CYP17A1), and the HSD3B1 into the direction of cortisol.

\section{Statistical analysis}

All results shown are mean \pm s.E.M. Comparison of group medians was carried out using the non-parametrical MannWhitney $U$ test for ranked data (SPSS/PC for Windows, 
A

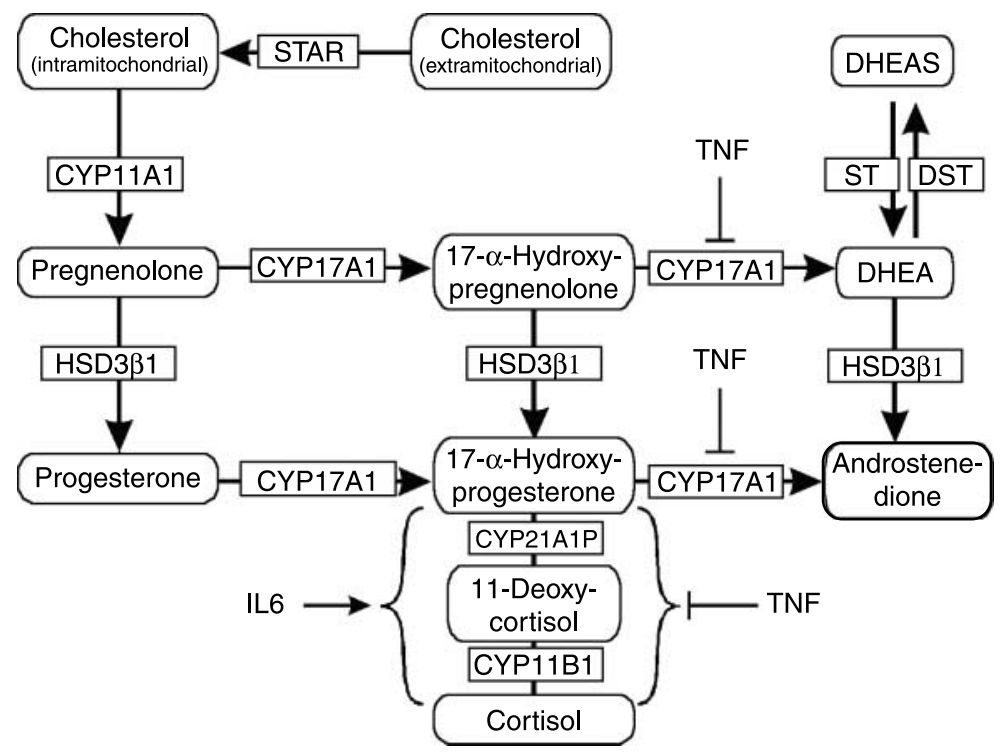

B Lean

Peripheral obese

Central obese

General obese
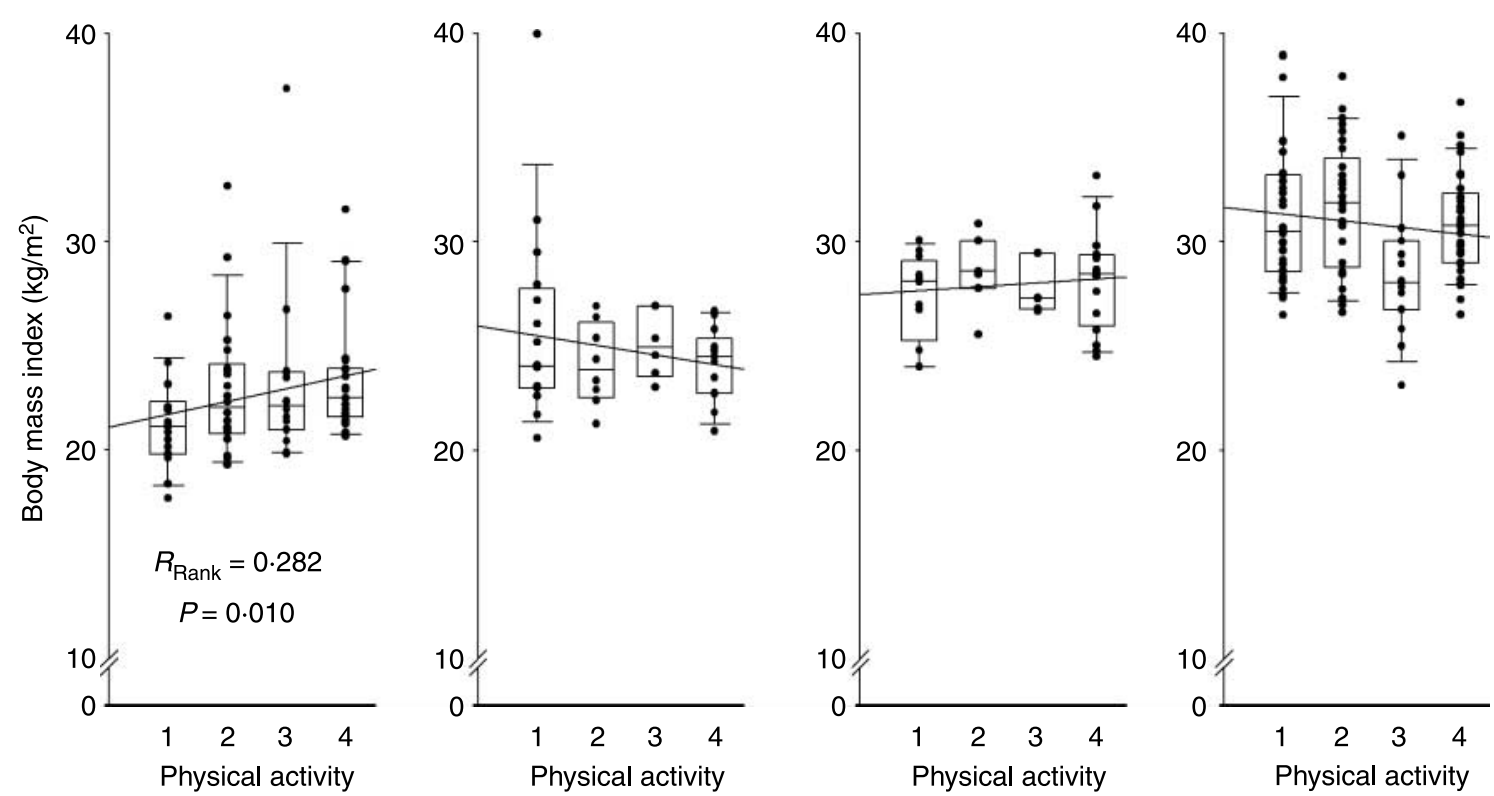

Figure 1 (A) Steroid cascade and (B) relation between physical activity and body mass index. (A) The adrenal steroid cascade with different conversion enzymes is demonstrated. In addition, it is indicated how cytokines can interfere with these enzymes. A line with a bar at the end represents an inhibitory influence whereas a line with an arrow indicates a stimulatory influence. The target hormones are cortisol, 17- $\alpha$-hydroxyprogesterone, dehydroepiandrosterone (DHEA), and androstenedione.

Abbreviations: HSD3B1, hydroxy- $\delta$-5-steroid dehydrogenase, $3 \beta$ - and steroid $\delta$-isomerase 1 ; DHEAS, DHEA sulfate; DST, DHEA sulfotransferase; CYP11B1, cytochrome P450, family 11, subfamily B, polypeptide 1; CYP17A1, cytochrome P450, family 17, subfamily A, polypeptide 1 and the 17/20-lyase; CYP21A1P, cytochrome P450, family 21, subfamily A, polypeptide 1 pseudogene; CYP11A1, cytochrome P450, family 11, subfamily A, polypeptide 1; ST, sulfatase; STAR, steroidogenic acute regulatory protein. (B) Relation between physical activity and body mass index in different obesity groups. Data are given as a black circle for every patient and as box plots with the 10th, 25th, 50th (median), 75th, and 90th percentile. If a significant relation existed, this is indicated by the Spearman rank correlation coefficient and its $P$ value. The line in the figure represents the linear regression line. Weekly physical activities are defined as follows: self-reported physical activity of at least $1 \mathrm{~h}$ duration per occasion (1: none/week, 2: once/week, 3: twice/week, 4: more than twice/week). 
V.12.0, SPSS, Inc., Chicago, IL, USA). Comparisons of several group medians were done with the Kruskal-Wallis test. Spearman rank correlation analysis was used to test the interrelation between two selected variables. Spearman rank correlation was used in order to minimize the effect of outliers. Differences were considered statistically significant, if $P<0 \cdot 05$.

\section{Results}

$B M I$ and $H O M A_{I R}$ index in different obesity and physical activity groups

Lean women with higher physical activity demonstrated increased BMI (Fig. 1B). Such an interrelation between physical activity and BMI was not observed in peripheral adipose, central adipose, and general obese women (Fig. 1B). From Fig. 1B, it is obvious that the median BMI was lowest in lean women followed by peripheral, central, and general obese women $(P<0 \cdot 001$ for the overall comparison, see also Supplementary Table 1). In addition, lean women demonstrated a low $\mathrm{HOMA}_{\mathrm{IR}}$ index, which was similar in peripheral obese women but markedly higher in central and general obese women $(P<0 \cdot 001$ for the overall comparison, Supplementary Table 1).

Plasma levels of cortisol, 17- $\alpha$-OHP, DHEA, and ASD in different obesity and physical activity groups

In the various groups, physical activity was neither related to plasma levels of cortisol nor to $17-\alpha-\mathrm{OHP}$ (Fig. 2A and B). However, the groups were different in plasma cortisol with highest values in peripheral adipose women followed by lean women, central adipose, or general obese women $(369 \pm 39$ vs $342 \pm 23$ vs $318 \pm 34$ vs $270 \pm 18 \mathrm{nmol} / 1, P=0 \cdot 035$ for the overall comparison). Similarly, plasma $17-\alpha-\mathrm{OHP}$ was highest in peripheral adipose women followed by lean women, central adipose, and general obese women $(3 \cdot 0 \pm 1 \cdot 5$ vs $1 \cdot 4 \pm$ $0 \cdot 1$ vs $1 \cdot 4 \pm 0 \cdot 1$ vs $1 \cdot 2 \pm 0 \cdot 1 \mathrm{nmol} / 1, P=0 \cdot 004$ for the overall comparison). This indicates that peripheral adipose women in comparison to other groups demonstrate a somewhat activated adrenal cortex.

In peripheral adipose women, physical activity was related to plasma levels of DHEA and ASD (Fig. 3A and B). Women with the highest physical activity demonstrated the highest plasma levels of these two hormones (Fig. 3A and B). A similar interrelation was not observed in the other groups (Fig. 3A and B). An overall comparison of all groups did not show a difference in plasma levels of DHEA and ASD (data not shown).

\section{Molar hormone ratios in different obesity and physical activity groups}

In order to demonstrate the predominance of an adrenal pathway, molar hormone ratios were analyzed. In peripheral

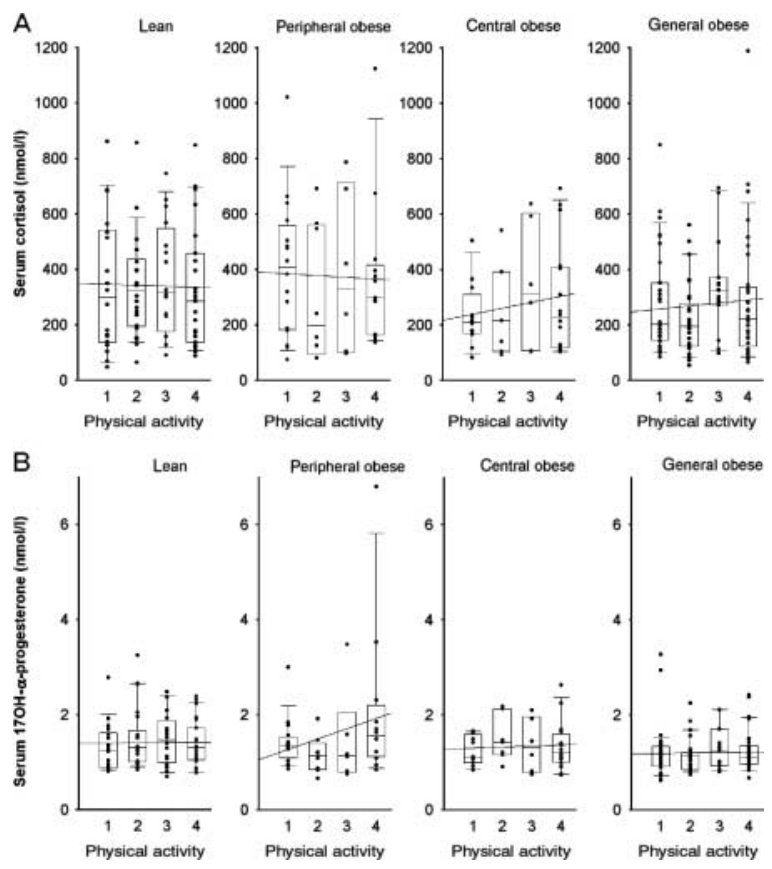

Figure 2 (A) Relation between physical activity and plasma levels of cortisol and (B) 17- $\alpha$-hydroxyprogesterone. In both parts of this figure, data are given as a black circle for every patient and as box plots with the 10th, 25th, 50th (median), 75th, and 90th percentile. The line in the figure represents the linear regression line. Different degrees of physical activity are defined in legend to Fig. 1.

Abbreviations: $17-\alpha$-OH-progesterone, 17- $\alpha$-hydroxyprogesterone.

adipose women, physical activity was negatively correlated with the ratio of plasma cortisol/plasma DHEA and plasma cortisol/plasma 17- $\alpha$-OHP (Fig. 4A and B). A similar interrelation was not observed in the other study groups (Fig. 4A and B). This indicates that in peripheral adipose women with high physical activity, the steroid hormone pathway is shifted to adrenal androgens and their precursor $17-\alpha$-OHP.

In order to better define the responsible enzyme step, we calculated the molar ratio ASD/17- $\alpha$-OHP for the 17,20lyase (second reaction of the CYP17A1, Fig. 1A) and the molar ratio of ASD/DHEA for the HSD3B1 (Fig. 1A). In neither of the groups were these two ratios related to physical activity (data not shown). In addition, these two ratios were not different between groups (data not shown).

\section{Molar hormone ratio of cortisol/DHEA and aortic calcification}

Since the ratio of plasma cortisol/plasma DHEA was low in peripheral adipose women with high physical activity, the interrelation between this particular ratio and aortic calcification was studied. It turned out that aortic calcification was related to elevated cortisol serum levels in relation to serum DHEA (Fig. 5A). This interesting phenomenon was only observed in peripheral adipose women (Fig. 5A). In other words, the higher the adrenal androgen in relation to cortisol the less was the severity of aortic calcification. 
A
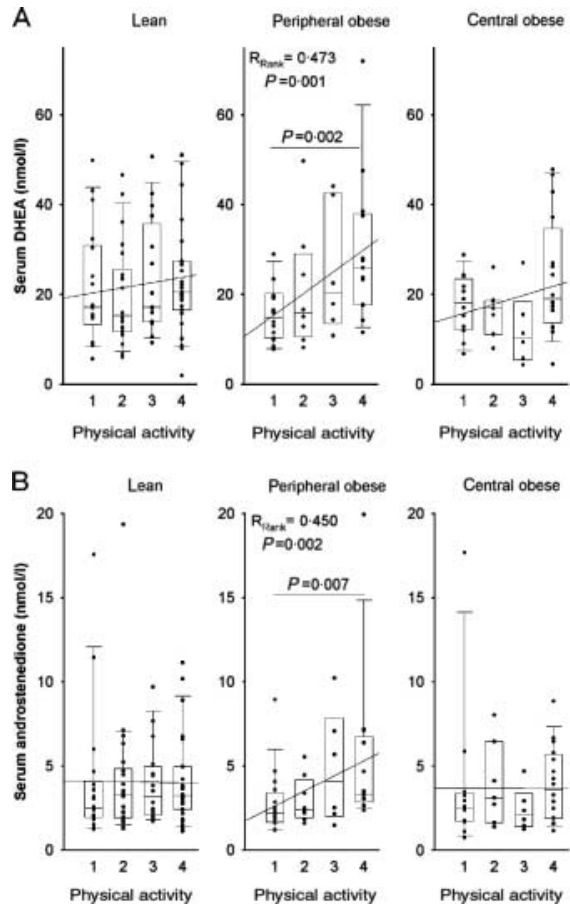

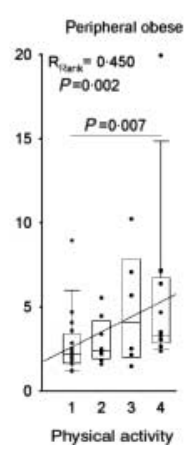

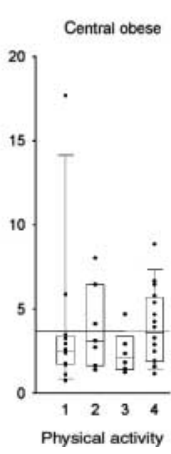

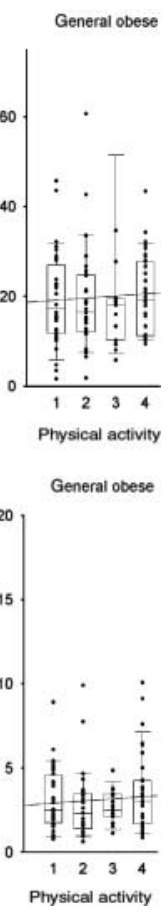

Physical activity

Figure 3 (A) Relation between physical activity and plasma levels of DHEA and (B) androstenedione. In both parts of this figure, data are given as a black circle for every patient and as box plots with the 10th, 25th, 50th (median), 75th, and 90th percentile. The line in the figure represents the linear regression line. Different degrees of physical activity are defined in legend to Fig. 1. If a significant relation existed, this is indicated by the Spearman rank correlation coefficient and its $P$ value. In addition, the $P$ value for the comparison of lean versus general obese women is given. Abbreviations: DHEA, dehydroepiandrosterone.

\section{Plasma levels of IL6 in different obesity and physical activity groups}

Since IL6 interferes with adrenal steroid hormone production (Ehrhart-Bornstein et al. 1998), it has been speculated to be involved as a mediator of physiological responses to exercise and obesity. In peripherally adipose women, physical activity was negatively correlated with plasma IL6 concentrations (Fig. 5B). Women with the highest physical activity demonstrated the lowest IL6 concentration. A similar interrelation was not observed in the other study groups. As expected, lean women demonstrated the lowest circulating IL6 concentration followed by peripheral adipose, central adipose, and general obese women $(2 \cdot 16 \pm 0 \cdot 17$ vs $2 \cdot 32 \pm$ $0 \cdot 22$ vs $2 \cdot 73 \pm 0 \cdot 38$ vs $3 \cdot 52 \pm 0 \cdot 29, P<0 \cdot 001)$.

\section{Discussion}

Distribution of body fat is an important factor in determining the risk for the insulin resistance syndrome and its sequelae to which peripheral adiposity confers protection (Tanko et al. 2003, Ferreira et al. 2004, Snijder et al. 2004). Distinction
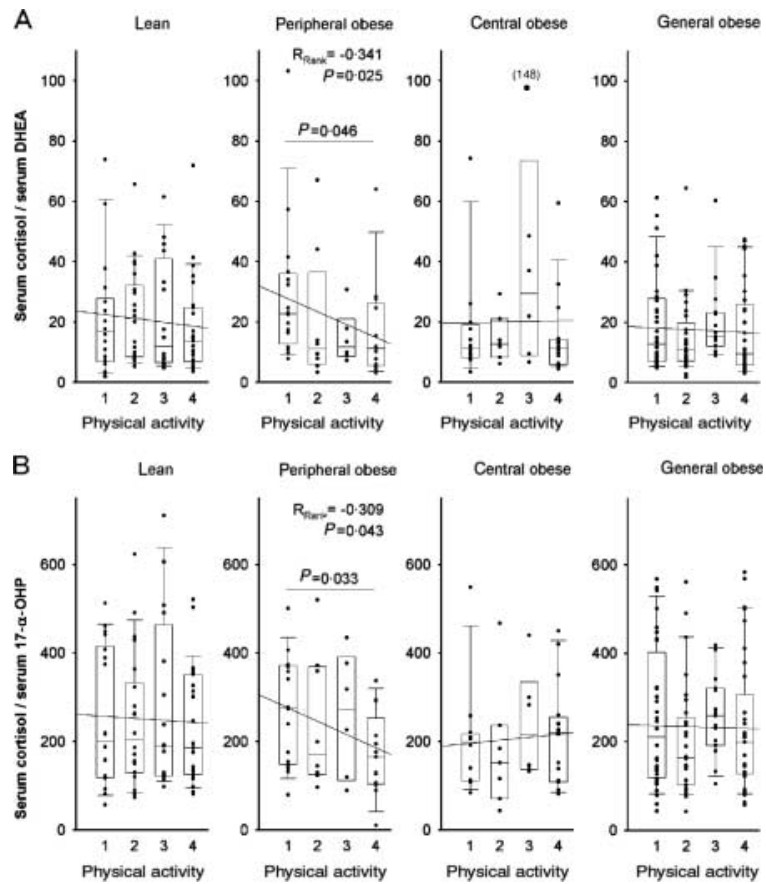

Figure 4 (A) Relation between physical activity and molar ratios of plasma cortisol/plasma DHEA and (B) plasma cortisol/plasma 17- $\alpha$ hydroxyprogesterone. Data are given as a black circle for every patient and as box plots with the 10th, 25th, 50th (median), 75th, and 90th percentile. The line in the figure represents the linear regression line. Different degrees of physical activity are defined in legend to Fig. 1. If a significant relation existed, this is indicated by the Spearman rank correlation coefficient and its $P$ value. In addition, the $P$ value for the comparison of lean versus general obese women is given. Abbreviations: $17-\alpha-\mathrm{OH}$-progesterone, 17- $\alpha$-hydroxyprogesterone; DHEA, dehydroepiandrosterone.

between peripheral versus central obesity in determining risk factors is based on epidemiological data but pathophysiologically relevant mechanisms are only partly identified. For the first time, this study integrated four different co-factors that all modulate insulin resistance and obesity-related illnesses: 1) physical activity, 2) fat distribution, 3) adrenal androgens, and 4) IL6 plasma levels. This study demonstrates that only in peripheral adipose elderly women, we can demonstrate a direct association between physical activity and increased circulating levels of adrenal androgens (in relation to cortisol), decreased plasma levels of IL6, and less severe aortic calcification. This clearly indicates that distinction of different states of adiposity need to be taken into account in the pathophysiology of obesity-related diseases.

As early as in 1986, the link between acute intense exercise and elevated serum levels of DHEA was reported in men (Cumming et al. 1986), which was later confirmed in women using DHEAS as a readout parameter (Keizer et al. 1987). Generally, it is important to distinguish between an exerciseinduced increase of serum DHEA in contrast to serum DHEAS because the activity of the sulfatase or DHEA sulfotransferase might be affected by training or by an altered 

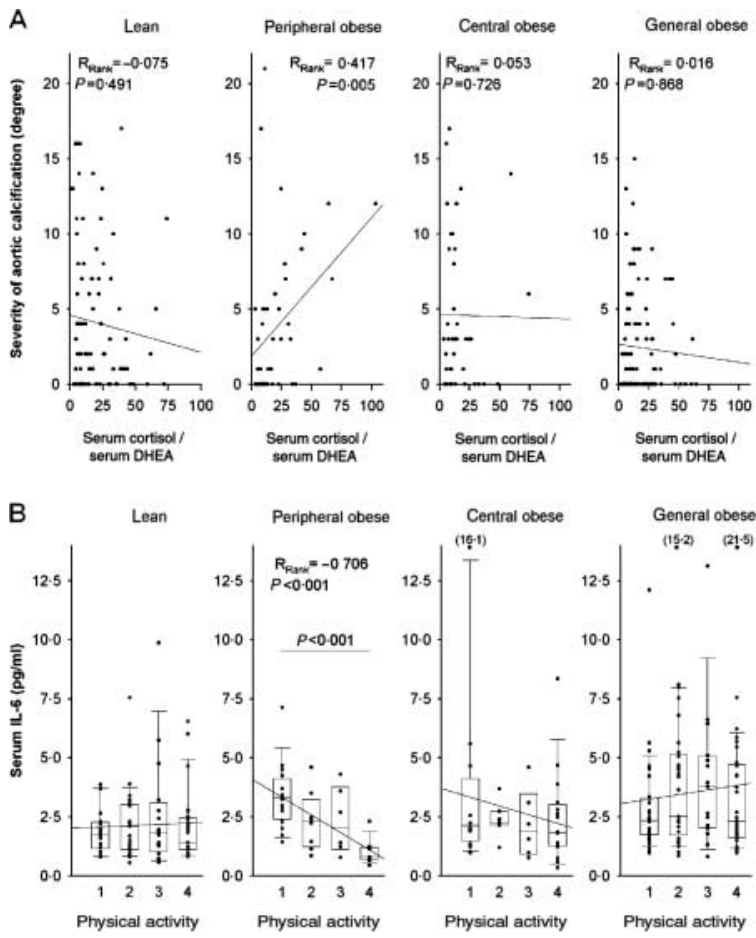

Figure 5 (A) Correlation between the molar ratio of plasma cortisol/plasma DHEA and severity of aortic calcification. The line in the figure represents the linear regression line. In addition, the Spearman rank correlation coefficient and its $P$ value are given. (B) Relation between physical activity and plasma interleukin (IL)6. Data are given as a black circle for every patient and as box plots with the 10th, 25th, 50th (median), 75th, and 90th percentile. The line in the figure represents the linear regression line. Different degrees of physical activity are defined in legend to Fig. 1. If a significant relation existed, this is indicated by the Spearman rank correlation coefficient and its $P$ value. In addition, the $P$ value for the comparison of lean versus general obese women is given.

sympathetic activity (Kizildere et al. 2003). An increase of DHEA is less pronounced in elderly people after exercise (Malarkey et al. 1993). However, findings of increased DHEA after exercise was not found in other studies in elderly women (Hersey et al. 1994, Milani et al. 1995, Abbasi et al. 1998, Hakkinen et al. 2000). Our present study indicates, that the discrepant findings might be explained by differences in body fat distribution, because only peripheral adipose women demonstrated the positive association between physical activity and plasma levels of DHEA and ASD. Body fat distribution has not been considered in the aforementioned exercise studies and, hence, stratification according to this important cofactor was not performed. It is presently unclear why exercise is able to increase plasma levels of DHEA and ASD but a feedback cross talk with exercise-induced IL6 secretion might play a role.

The impact of exercise on plasma IL6 is biphasic characterized by an acute increase and longer term decrease: thus, strenuous exercise increases serum levels of IL6 (Pedersen et al. 2001), which might be an important factor to stimulate the adrenal production of cortisol and adrenal androgens (Fig. 1A). However, it has been shown that long term increase in exercise reduces plasma IL6 levels in postmenopausal obese women (Ryan \& Nicklas 2004). Ryan et al. proposed the existence of a causal link between IL6 mediated low grade inflammation and insulin resistance as IL6 was an independent predictor of insulin sensitivity. Thus, muscle-derived IL6 might be considered as a secreted hormone supporting the adrenal cortex (Pedersen et al. 2001). On the other hand, adrenal androgens such as DHEA and ASD inhibit secretion of IL6 (Straub et al. 1998, Kipper-Galperin et al. 1999, Gordon et al. 2001, Kim et al. 2006). These two elements might be viewed as a negative feedback hormonal loop. It is interesting that such a negative feedback cross talk during long-term exercise can lead to lower levels of IL6 (our study in peripheral obese women and refs (Colbert et al. 2004, Jankord \& Jemiolo 2004, Kohut et al. 2006)). Such mechanism could in part explain the decrease in IL6 plasma levels often associated with continued elevation of exercise levels. However, the accompanying increase in skeletal muscle IL6 receptor expression seen in subjects improving aerobic capacity as a consequence of elevated exercise could also explain a compensatory decrease in plasma IL6 as the simple consequence of improved muscle IL6 sensitivity (Keller et al. 2005). We propose that continuous long-term exercise gradually increases adrenal androgens, which then might exert an inhibitory influence on IL6 secretion from leukocytes, myocytes, and other sources. In elderly people between 70 and 79 years, self-reported levels of physical activity is inversely correlated with plasma levels of IL6 (Colbert et al. 2004), and no attempts were made to differentiate these subjects by anthropometric measures. Thus, it came as a surprise that we only observed this phenomenon in peripherally adipose women, the reasons for which are presently not known. One might speculate that increased plasma levels of fat-derived IL6 in central and general obese women are too high to institute the abovementioned negative feedback loop.

Using molar hormone ratios, the preponderance of DHEA in relation to cortisol was evident. In addition, the molar ratio of plasma cortisol/plasma 17-OHP indicates that one part of this effect could be due to a change on the level of the CYP21A1P and CYP11B1 (Fig. 1A). Since this pathway is stimulated by IL6 (Ehrhart-Bornstein et al. 1998), a long-term decrease of IL6 might shift the pathway predominance from cortisol to adrenal androgens. Interestingly, the CYP17A1 (ASD/17-OHP ratio) and the HSD3B1 (ASD/DHEA ratio) do not seem to be involved because these ratios were not associated with physical activity in all study groups. Only in peripheral adipose women, we suggest that long-term exercise can influence the CYP21A1P and CYP11B1 leading to increased androgens, which help to decrease IL6 so that these two enzymes are not further stimulated by IL6 (favorable circle). Peripheral adipose women, but not central or general obese women might have an optimum constellation where the favorable circle is maintained or switched on. Since IL6 
is an important factor for insulin resistance syndrome, atherosclerosis and related sequelae (Fernandez-Real \& Ricart 2003), exercise-induced reduction of IL6 in peripheral adipose women seems to confer a protective effect.

Importantly, in peripheral adipose women, the ratio of serum cortisol/serum DHEA was positively related to the severity of aortic calcification, which indicates that high androgen levels in relation to cortisol probably exerts a beneficial effect in this group. A similar positive correlation was not observed in lean, central adipose, and general adipose women. We do not know whether, or not, this effect is mediated through inhibition of IL6 secretion. However, since IL6 was found to be related to an increased severity of atherosclerosis, androgen-induced inhibition of IL6 secretion might be a relevant factor.

In conclusion, in peripheral adipose women, physical activity was related to increased plasma androgens and relatively lower plasma cortisol, which was accompanied by lower plasma levels of IL6 and less sever aortic calcification. This positive effect of long-term exercise was not observed in central and general obese women. Thus, particularly peripheral adipose women might profit from continuous moderate exercise leading to a decreased risk for insulin resistance syndrome and its negative sequelae. This study provides evidence for a possible new mechanism underlying this protective effect.

\section{Declaration of Interest}

The authors declare that there are no conflicts of interest.

\section{Funding}

This study was supported by the respective institutions.

\section{References}

Abbasi A, Duthie EH Jr, Sheldahl L, Wilson C, Sasse E, Rudman I \& Mattson DE 1998 Association of dehydroepiandrosterone sulfate, body composition, and physical fitness in independent community-dwelling older men and women. Journal of the American Geriatrics Society 46 263-273.

Anderson DC \& Yen SS 1976 Effects of estrogens on adrenal 3 betahydroxysteroid dehydrogenase in ovariectomized women. Journal of Clinical Endocrinology and Metabolism 43 561-570.

Barone RM, Shamonki IM, Siiteri PK \& Judd HL 1979 Inhibition of peripheral aromatization of androstenedione to estrone in postmenopausal women with breast cancer using delta 1-testololactone. Journal of Clinical Endocrinology and Metabolism 49 672-676.

Colbert LH, Visser M, Simonsick EM, Tracy RP, Newman AB, Kritchevsky SB, Pahor M, Taaffe DR, Brach J, Rubin S et al. 2004 Physical activity, exercise, and inflammatory markers in older adults: findings from the Health, Aging and Body Composition Study. Journal of the American Geriatrics Society 52 1098-1104.

Cumming DC, Brunsting LA III, Strich G, Ries AL \& Rebar RW 1986 Reproductive hormone increases in response to acute exercise in men. Medicine and Science in Sports and Exercise 18 369-373.
Ehrhart-Bornstein M, Hinson JP, Bornstein SR, Scherbaum WA \& Vinson GP 1998 Intraadrenal interactions in the regulation of adrenocortical steroidogenesis. Endocrine Reviews 19 101-143.

Fernandez-Real JM \& Ricart W 2003 Insulin resistance and chronic cardiovascular inflammatory syndrome. Endocrine Reviews 24 278-301.

Ferreira I, Snijder MB, Twisk JW, van MW, Kemper HC, Seidell JC \& Stehouwer CD 2004 Central fat mass versus peripheral fat and lean mass: opposite (adverse versus favorable) associations with arterial stiffness? The Amsterdam Growth and Health Longitudinal Study Journal of Clinical Endocrinology and Metabolism 89 2632-2639.

Gordon CM, LeBoff MS \& Glowacki J 2001 Adrenal and gonadal steroids inhibit IL-6 secretion by human marrow cells. Cytokine 16 178-186.

Grant RW \& Meigs JB 2004 Should the insulin resistance syndrome be treated in the elderly? Drugs \& Aging 21 141-151.

Hakkinen K, Pakarinen A, Kraemer WJ, Newton RU \& Alen M 2000 Basal concentrations and acute responses of serum hormones and strength development during heavy resistance training in middle-aged and elderly men and women. Journals of Gerontology. Series A, Biological Sciences and Medical Sciences 55 B95-B105.

Hautanen A, Manttari M, Manninen V, Tenkanen L, Huttunen JK, Frick MH \& Adlercreutz H 1994 Adrenal androgens and testosterone as coronary risk factors in the Helsinki Heart Study. Atherosclerosis 105 191-200.

Hersey WC III, Graves JE, Pollock ML, Gingerich R, Shireman RB, Heath GW, Spierto F, McCole SD \& Hagberg JM 1994 Endurance exercise training improves body composition and plasma insulin responses in 70- to 79-year-old men and women. Metabolism 43 847-854.

Jankord R \& Jemiolo B 2004 Influence of physical activity on serum IL-6 and IL-10 levels in healthy older men. Medicine and Science in Sports and Exercise 36 960-964.

Kauppila LI, Polak JF, Cupples LA, Hannan MT, Kiel DP \& Wilson PW 1997 New indices to classify location, severity and progression of calcific lesions in the abdominal aorta: a 25-year follow-up study. Atherosclerosis 132 245-250.

Keizer HA, Kuipers H, de HJ, Beckers E \& Habets L 1987 Multiple hormonal responses to physical exercise in eumenorrheic trained and untrained women. International Journal of Sports Medicine 8 139-150.

Keller C, Steensberg A, Hansen AK, Fischer CP, Plomgaard P \& Pedersen BK 2005 Effect of exercise, training, and glycogen availability on IL-6 receptor expression in human skeletal muscle. Journal of Applied Physiology 99 20752079.

Kim SK, Shin MS, Jung BK, Shim JY, Won HS, Lee PR \& Kim A 2006 Effect of dehydroepiandrosterone on lipopolysaccharide-induced interleukin-6 production in DH82 cultured canine macrophage cells. Journal of Reproductive Immunology 70 71-81.

Kipper-Galperin M, Galilly R, Danenberg HD \& Brenner T 1999 Dehydroepiandrosterone selectively inhibits production of tumor necrosis factor alpha and interleukin-6 correction of interlukin-6 in astrocytes. International Journal of Developmental Neuroscience 17 765-775.

Kizildere S, Glück T, Zietz B, Schölmerich J \& Straub RH 2003 During a corticotropin-releasing hormone test in healthy subjects, administration of a beta-adrenergic antagonist induced secretion of cortisol and dehydroepiandrosterone sulfate and inhibited secretion of ACTH. European Journal of Endocrinology 148 45-53.

Kohut ML, McCann DA, Russell DW, Konopka DN, Cunnick JE, Franke WD, Castillo MC, Reighard AE \& Vanderah E 2006 Aerobic exercise, but not flexibility/resistance exercise, reduces serum IL-18, CRP, and IL-6 independent of beta-blockers, BMI, and psychosocial factors in older adults. Brain, Behavior, and Immunity 20 201-209.

Laukkanen JA, Kurl S \& Salonen JT 2002 Cardiorespiratory fitness and physical activity as risk predictors of future atherosclerotic cardiovascular diseases. Current Atherosclerosis Reports 4 468-476.

Malarkey WB, Hall JC, Rice RR Jr, O’Toole ML, Douglas PS, Demers LM \& Glaser R 1993 The influence of age on endocrine responses to ultraendurance stress. Journal of Gerontology 48 M134-M139.

Milani RV, Lavie CJ, Barbee RW \& Littman AB 1995 Lack of effect of exercise training on dehydroepiandrosterone-sulfate. American Journal of the Medical Sciences 310 242-246. 
Nestler JE, Clore JN \& Blackard WG 1992 Dehydroepiandrosterone: the 'missing link' between hyperinsulinemia and atherosclerosis? FASEB Journal 6 3073-3075.

Pedersen BK, Steensberg A \& Schjerling P 2001 Muscle-derived interleukin6: possible biological effects. Journal of Physiology 536 329-337.

Phillips GB, Pinkernell BH \& Jing TY 1997 Relationship between serum sex hormones and coronary artery disease in postmenopausal women. Arteriosclerosis, Thrombosis, and Vascular Biology 17 695-701.

Raji A \& Plutzky J 2002 Insulin resistance, diabetes, and atherosclerosis: thiazolidinediones as therapeutic interventions. Current Cardiolog $\gamma$ Reports 4 514-521.

Ryan AS \& Nicklas BJ 2004 Reductions in plasma cytokine levels with weight loss improve insulin sensitivity in overweight and obese postmenopausal women. Diabetes Care 27 1699-1705.

Snijder MB, Henry RM, Visser M, Dekker JM, Seidell JC, Ferreira I, Bouter LM, Yudkin JS, Westerhof N \& Stehouwer CD 2004 Regional body composition as a determinant of arterial stiffness in the elderly: the Hoorn Study. Journal of Hypertension 22 2339-2347.

Straub RH, Konecna L, Hrach S, Rothe G, Kreutz M, Schölmerich J, Falk W \& Lang B 1998 Serum dehydroepiandrosterone (DHEA) and DHEA sulfate are negatively correlated with serum interleukin-6 (IL-6), and DHEA inhibits IL-6 secretion from mononuclear cells in man in vitro: possible link between endocrinosenescence and immunosenescence. Journal of Clinical Endocrinology and Metabolism 83 2012-2017.

Straub RH, Pongratz G, Schölmerich J, Kees F, Schaible TF, Antoni C, Kalden JR \& Lorenz HM 2003 Long-term anti-tumor necrosis factor antibody therapy in rheumatoid arthritis patients sensitizes the pituitary gland and favors adrenal androgen secretion. Arthritis and Rheumatism 48 1504-1512.
Tanko LB, Bagger YZ, Alexandersen P, Larsen PJ \& Christiansen C 2003 Peripheral adiposity exhibits an independent dominant antiatherogenic effect in elderly women. Circulation 107 1626-1631.

Tanko LB, Bruun JM, Alexandersen P, Bagger YZ, Richelsen B, Christiansen C \& Larsen PJ 2004 Novel associations between bioavailable estradiol and adipokines in elderly women with different phenotypes of obesity: implications for atherogenesis. Circulation 110 2246-2252.

Tanko LB, Bagger YZ, Qin G, Alexandersen P, Larsen PJ \& Christiansen C 2005 Enlarged waist combined with elevated triglycerides is a strong predictor of accelerated atherogenesis and related cardiovascular mortality in postmenopausal women. Circulation 111 1883-1890.

Vicennati V \& Pasquali R 2000 Abnormalities of the hypothalamic-pituitaryadrenal axis in nondepressed women with abdominal obesity and relations with insulin resistance: evidence for a central and a peripheral alteration. Journal of Clinical Endocrinology and Metabolism 85 4093-4098.

Wilson PW, Kauppila LI, O’Donnell CJ, Kiel DP, Hannan M, Polak JM \& Cupples LA 2001 Abdominal aortic calcific deposits are an important predictor of vascular morbidity and mortality. Circulation 103 1529-1534.

Wu FC \& von EA 2003 Androgens and coronary artery disease. Endocrine Reviews 24 183-217.

Received in final form 2 July 2008

Accepted 10 July 2008

Made available online as an Accepted Preprint 10 July 2008 